Review began 12/07/2021 Review ended 12/17/2021 Published 01/02/2022

(๑) Copyright 2022

Schaffer et al. This is an open acces article distributed under the terms of the Creative Commons Attribution License CCBY 4.0., which permits unrestricted use, distribution, and reproduction in any medium, provided the original author and source are credited.

\section{Community-Based, Rapid HIV Screening and Pre- Exposure Prophylaxis Initiation: Findings From a Pilot Program}

\author{
David H. Schaffer ${ }^{1}$, Lindsey M. Sawczuk ${ }^{2}$, Hui Zheng ${ }^{1}$, Wendy L. Macias-Konstantopoulos ${ }^{3,1}$
}

1. Department of Emergency Medicine, Massachusetts General Hospital, Boston, USA 2. Department of Medicine, Tulane University School of Medicine, New Orleans, USA 3. Department of Emergency Medicine, Harvard Medical School, Boston, USA

Corresponding author: David H. Schaffer, dschafferem@gmail.com

\section{Abstract}

\section{Objective}

Many individuals do not have regular access to medical care and preventative health services, suggesting the need for alternative access to HIV testing and pre-exposure prophylaxis (PrEP). The purpose of this study is to describe a novel, community-based HIV screening, a PrEP initiation program, and report preliminary findings.

\section{Methods}

One Tent Health, a 501(c)(3) nonprofit organization, launched a pop-up HIV screening and PrEP initiation program in high-risk areas of Washington, DC in 2017. We describe the unique features of the program and report 25 months of screening, risk assessment, and PrEP education data. Odds ratios were calculated to identify disparities in both HIV risk factors and prior HIV testing.

\section{Results}

Between October 2017 and November 2019, 846 individuals underwent HIV screening. Six individuals (0.709\%) screened HIV-positive. Approximately 13\% had never been screened for HIV, and another 13\% had at least one major risk factor for HIV. Individuals who self-identified as White were more likely to have risk factors (OR 2.19, $\mathrm{p}=0.0170$ ) and less likely to have ever been tested (OR 0.50, p = 0.0409). Individuals who self-identified as Black or African American were less likely to have risk factors for HIV (OR 0.57, p = 0.0178). Disparities by sex and gender were also observed.

\section{Conclusions}

This program appears to be the first of its kind within the United States. We found the program to be costeffective, well-received by the community, and accessible by high-risk and unreached populations while further revealing the role of race and gender in the HIV epidemic.

Categories: HIV/AIDS, Public Health, Epidemiology/Public Health

Keywords: pre-exposure prophylaxis (prep), community-based, linkage to care, community health research, hiv testing

\section{Introduction}

Early diagnosis and treatment remain key components of reducing transmission and improving the health of people living with HIV (PLWH) [1]. In the United States (US), ongoing localized epidemics require innovative disease burden reduction strategies. Washington, DC has the highest incidence and prevalence rates of HIV infection as compared to all states in the US [2]. Prevalence estimates indicate approximately $2 \%-3 \%$ of the DC population are PLWH, potentially double or triple the WHO threshold for epidemic status [2-4]. Further, the incidence of new HIV cases in DC did not decline between 2014 - 2018 [4].

The history of this localized epidemic is complex, though it is clear that HIV rates correlate with factors such as income, racial background, and sexual partners [5-6]. Governmental programs focused on risk reduction have demonstrated uptake; for example, the DC Department of Health reported that in 2016 over five million male and female condoms were distributed, while nearly one million used needles were collected [7]. Yet, gaps in HIV prevention persist; nearly $25 \%$ of those who screened HIV-positive remained without linkage to care (LTC) within three months of screening [5]. Further, the lifetime risk of acquiring HIV remains as high as one in 13 in DC compared to about one in 100 for the nation as a whole [8]. Ideally, all residents aged 15 to 65 would receive their recommended HIV screening once and subsequent tests as dictated by lifestyle risk factors [9].

Many individuals at high risk for HIV infection do not have regular access to medical care and preventative 
health services, thus suggesting a need for expanded testing programs [10]. Community-based screening programs in a wide range of settings have previously been explored as a means to address this problem [1114]. These programs, including mobile, door-to-door, school-based, and other non-facility-based screening, have led to earlier diagnosis, higher average $\mathrm{CD} 4$ count at the time of diagnosis, and a higher intake of patients never screened before [13]. In urban settings in the US, those at high risk for HIV were more likely to seek care in these non-traditional screening options [15]. Community-based programs also reported equivalent LTC parameters as compared to facility-based centers [13]. These findings demonstrate the utility of community-based screening options, particularly in high-risk areas.

While community-based programs have many significant advantages, they may also be difficult to maintain. In Cape Town, South Africa, mobile HIV screening was found to be particularly cost-effective, though, ultimately, total operating costs neared \$1 million (USD) over a two-year period, which may not be feasible for many healthcare organizations [16]. Implementation of a mobile unit for HIV screening may also be susceptible to other obstacles beyond financial costs, such as licensing, insurance, staff training, and physical space for both parking and screening sessions.

The present analysis introduces a new screening program designed to minimize the barriers to communitybased screening while reaching high-risk populations. This rapid, pop-up HIV screening and pre-exposure prophylaxis (PrEP) initiation program in Washington, DC was launched by One Tent Health (OTH), a 501(c) (3) nonprofit, and began screenings in October 2017. We describe the program below and present an analysis of preliminary data. This pop-up screening model was implemented with the following intentions:

1. Provide free, fast, HIV screening and LTC, regardless of insurance status

2. Educate clients about PrEP and provide rapid prescriptions when eligible

3. Minimize the socioeconomic, temporospatial, and health literacy barriers that reduce access to HIV screening and PrEP prescription

4. Reduce the stigma surrounding HIV, HIV screening, and PrEP

\section{Materials And Methods}

OTH enrolled over 1,600 volunteers predominantly from universities in the Washington, DC area. No healthcare professionals were deployed as sessions included only screening tests waived under the Clinical Laboratory Improvement Amendments of 1988 (CLIA). Areas with high HIV prevalence but without established HIV screening clinics were selected as screening sites. Given the significant burdens of using a mobile van for testing, OTH raised a 10’ x 10' canvas tent in high foot-traffic areas within these disproportionately affected neighborhoods, typically outside of grocery stores, pharmacies, or laundromats. Five trained volunteers conducted CLIA-waived HIV screening with the INSTI ${ }^{\circledR}$ HIV-1/HIV-2 Rapid Antibody Kit (bioLytical ${ }^{\circledR}$, Richmond, BC, Canada), a fingerstick kit that shows results in approximately 60 seconds. Volunteers underwent training designed to prepare them with the knowledge and skills necessary to conduct PrEP and HIV education, assess risk factors, discuss results, and a link to care. Volunteers were assigned distinct roles to allow for leadership, outreach, intake, screening, and education.

Upon intake, clients completed a tablet-facilitated demographics questionnaire and assessment of risk factors, prior HIV testing, and knowledge or use of PrEP. Submission of this form electronically relayed the information to the screening volunteer inside the screening tent. PrEP education was conducted and the client then entered the tent and underwent screening. Post-screening disposition was determined by screening results and indications for PrEP initiation. The screening process took approximately 10 - 15 minutes from registration to disposition. LTC was coordinated with clinical partners off-site. When PrEP was indicated, clients who screened negative were able to meet with a clinical partner on-site to review medical history and risk factors and undergo rapid initiation of PrEP. Clients were asked to complete postscreening surveys regarding their experience.

Clients who screened positive were informed of their results, the need for confirmatory testing, and the importance of initiating care. OTH prioritized same-day confirmatory testing via clinical partners in Washington, DC since same-day confirmation has been associated with accelerated LTC [17]. Clients who declined immediate referrals were offered other options for LTC. All positive screening results were reported to the DC Department of Health to facilitate further outreach and disease surveillance. Follow-up for those clients who screened positive were conducted within one week of screening and again at approximately one year.

\section{Data collection}

Client data was collected by OTH during intake and screening and subsequently updated to reflect follow-up and LTC results. De-identified, retrospective data from a two-year screening period were provided by OTH for analysis. Data included screening dates and locations, client demographic information, HIV risk factors, and HIV screening results. Results of an anonymous post-screening satisfaction survey were also provided. The Mass General Brigham Institutional Review Board recommended this study (\#2019P003138) as non- 


\section{Cureus}

human subjects research exempt from continuing review.

\section{Data analysis}

Descriptive statistics are presented as frequencies and percentages for categorical variables and as means and standard deviations for continuous variables. Bivariate analyses were used to explore the relationships between client characteristics (race/ethnicity and sex/gender) and the presence of HIV risk factors and previous HIV testing. Pearson's Chi-Square was used to determine significance, except when the sample size was prohibitively small, in which case Fisher's exact t-test was used. Statistical significance was defined as p $<0.05$. Odds ratios were calculated using open-source software via Vassar College at vassarstats.net.

Few assumptions were made in preparation for data analysis. All data were self-reported, except for HIV screening results. A review of the data indicated several repeat clients. A small minority of clients were either unsure or chose not to report if they had ever been tested for HIV, in which case they were considered to have never been tested. Similarly, very few clients did not respond to questions about race, which were added to the "prefer not to say" category. In all other instances of missing data, the variable was excluded from the analysis.

\section{Role of funding source}

There were no sponsors or funding for this study.

\section{Results}

A total of 846 individuals underwent HIV screening across 72 screening sessions between October 2017 and November 2019 in Wards 4 through 8 of Washington, DC (Table 1). Participants were an average age of 41.7 years, of near equal sex distribution, and from a range of racial backgrounds. Nearly $96 \%$ of clients completed HIV screening after registration. Among these, six individuals screened positive for HIV (Table 2). Two were known positives who indicated they were linked to care and adherent to antiretroviral therapy, while four individuals were new preliminary positives. Approximately $13 \%$ of clients had never been tested for HIV. Individuals who self-identified as White were less likely to have ever been tested for HIV than were those of other racial backgrounds (OR 0.50, $p=0.04$ ), and cisgender men were less likely to have ever been tested for HIV than were those of other gender identities (OR 0.53, $p=0.0027$ ).

Patient Characteristics

Age

Age, mean

Age, median

Biological Sex, \%, Gender Identity

Male sex, $51.5 \%$

Female sex, $48.5 \%$

Race

Black or African American

White or Caucasian

Native American or Alaskan Native

Asian or Pacific Islander

Other

Prefer not to say

Ethnicity

Hispanic or Latinx

Sexuality

Estimated \% of male clients who identify as MSM
$4.37 \%$

Proportion (\%, unless otherwise specified)

41.7 years

39 years

$51.5 \%$ Cis male

$0.00 \%$ Transgender female

$48.01 \%$ Cis female

$0.49 \%$ Transgender male

$82.39 \%$

$6.62 \%$

$1.06 \%$

$1.54 \%$

$6.15 \%$

$2.25 \%$

$3 \%$

Previous HIV Testing 


\section{Cureus}

\begin{tabular}{ll} 
Yes & $83.69 \%$ \\
No & $13.59 \%$ \\
Unsure & $1.65 \%$ \\
Prefer not to say & $1.06 \%$ \\
Negious HIV Test Result & \\
Positive & $81.21 \%$ \\
Unsure & $0.71 \%$ \\
Not applicable & $3.66 \%$ \\
Prefer not to say & $13.48 \%$ \\
Major Risk Factors for HIV & $0.95 \%$ \\
At least 1 major risk factor & \\
\hline At least 2 major risk factors & $12.88 \%$ \\
IV drug use & $3.78 \%$ \\
Sharing of needles (among IV drug users) & $3.48 \%$ \\
A partner who is HIV+ & $13.79 \%$ \\
Piercing with an unsterilized needle & $2.96 \%$ \\
Engage in sex work & $3.31 \%$ \\
History of hepatitis B or C & $5.08 \%$ \\
\hline
\end{tabular}

TABLE 1: Demographics, Risk Factors, and Prior HIV Testing

MSM: men who have sex with men

\begin{tabular}{|l|l|}
\hline \hline Screening result & $\%$ of $\mathbf{n}=846$ \\
\hline Reactive (preliminary positive) & $0.71 \%$ \\
\hline Non-reactive (presumed negative) & $99.29 \%$ \\
\hline Did not undergo screening after registration & $4.19 \%$ \\
\hline New positives & $0.47 \%$ \\
\hline PrEP Education and Linkage & $\%$ of $\mathbf{n}=644$ \\
\hline Heard of PrEP & $29.51 \%$ \\
Currently taking PrEP & $0.88 \%$ \\
\hline Requested linkage to a PrEP provider & $29.19 \%$ \\
\hline Requested same-day PrEP prescription & $27.36 \%$ \\
\hline
\end{tabular}

\section{TABLE 2: HIV Screening and PrEP Results with One Tent Health}

PrEP: pre-exposure prophylaxis

In addition to previous HIV testing, clients were assessed for HIV risk factors and counseled on riskreduction strategies (Table 3). HIV risk factors included injection drug use with or without needle-sharing, HIV-positive sexual partner(s), body piercing with unsterilized needles, commercial sex acts (sex in exchange for money, drugs, or anything of value), and history of hepatitis B or C. Nearly $13 \%$ of clients reported at least one of these risk factors and the prevalence of individual risk factors ranged from approximately $3 \%$ to 


\section{Cureus}

$5 \%$, the most common of which was an engagement in commercial sex acts. Clients with HIV risk factors were no more or less likely to have ever been screened for HIV than those without risk factors (OR 1.89, 95\% CI 0.93 - 3.86). Approximately $6.8 \%$ of all clients were men who have sex with men (MSM) (13\% of all cisgender male clients); as limited data was available on sexual partners, sexual orientation was not considered a risk factor here. Similarly, although $61 \%$ of clients reported ever engaging in condomless sex, this was not considered a risk factor due to limited information regarding partners, relationships, and instances of condomless sex. Individuals who self-identified as Black or African American were less likely than individuals of other racial backgrounds to have risk factors for HIV (OR 0.57, p = 0.018), while individuals who self-identified as White were more likely to have risk factors for HIV (OR 2.19, $\mathrm{p}=0.0170$ ). With regards to sex and gender, which were self-reported separately, cisgender men were more likely to report risk factors for HIV (OR 1.57, $\mathrm{p}=0.029)$, while cisgender women were less likely to report risk factors for HIV (OR 0.53, p = 0.0028).

Race, Ethnicity, or Gender
Race and Ethnicity
Black or African American
White
Native American or Alaska Native
Hispanic or Latinx
Sex and Gender
Male
Female
Translander
Transgender male
Race and Ethnicity
Black or African American
White

$$
\begin{aligned}
& \text { Odds Ratio }(\mathrm{Cl}), \mathrm{P} \text {-Value } \\
& \text { Odds of having risk factors for HIV } \\
& 0.57(0.36-0.91), p=0.0178 \\
& 2.19(1.13-4.21), p=0.0170 \\
& 0.00(\mathrm{~N} / \mathrm{A}), p=0.3849 \\
& 0.56(0.07-4.35), p=0.7106 \\
& 0.81(0.24-2.72), p=1.0000
\end{aligned}
$$

Odds of having risk factors for HIV

$$
\begin{aligned}
& 1.57(1.04-2.37), p=0.0296 \\
& 0.53(0.35-0.81), p=0.0028 \\
& \infty(N / A), p=0.1288
\end{aligned}
$$

Odds of ever receiving prior HIV test

$1.07(0.63-1.80), p=0.8065$

$0.50(0.25-0.98), p=0.0409$

$1.27(0.16-10.24), p=1.0000$

$0.31(0.09-1.04), p=0.0680$

$1.33(0.40-4.48), p=0.7863$

Odds of ever receiving a prior HIV test

$0.53(0.35-0.81), p=0.0027$

$1.67(1.10-2.51), p=0.0146$

$\infty(\mathrm{N} / \mathrm{A}), \mathrm{p}=1.0000$

TABLE 3: Odds of Risk Factors for HIV or Prior Testing by Race, Ethnicity, and Gender, as Compared to all Other Races, Ethnicities, or Genders

N/A: not available

PrEP education occurred during the intake of 644 clients (Table 2). Over $70 \%$ of clients had never heard of PrEP, less than $1 \%$ were using PrEP, and approximately $27 \%$ - $29 \%$ of clients requested either PrEP prescription or linkage to another PrEP provider.

Of the 169 clients who participated in the post-screening survey, 95.3\% reported they were "very pleased" with their experience and $95.8 \%$ reported the process took 15 minutes or less. None rated their experience at less than 4 out of 5 on a Likert scale.

\section{Discussion}

Community-based HIV screening offers access to care for individuals who may typically be missed by the healthcare system and allows providers to meet patients where they are, regardless of time, geography, 
health literacy, or financial barriers. Others have found success around the world with mobile HIV screening in a variety of settings [13, 15-16]. Herein, we describe a unique pop-up screening program, designed to provide rapid, free HIV screening and PrEP education in high-risk areas while minimizing both barriers to access and associated stigma.

\section{Population reached}

This screening program was well-received by the community, with 846 clients undergoing HIV screening and PrEP education, and post-screening survey results indicating 95.3\% were "very pleased" with their experience. Clients screened for HIV represented approximately $96 \%$ of those who completed intake, further suggesting the feasibility of the model and acceptability among clients. Nearly $13 \%$ of clients had at least one major risk factor for HIV, and another $13 \%$ had never been screened for HIV. Neither identification as MSM (13\%) nor report of condomless sex (61\%) was considered risk factors due to the limited data on partners, relationships, or instances of condomless sex.

The data suggest that pop-up screening reached a high-risk population. HIV surveillance indicated that the highest risk age groups were 35 - 44 and 45 - 55 years of age [4], a range that comprised approximately $40 \%$ of the OTH clients. The racial group historically felt to be at the highest risk, Black or African-American [4, 8], was well-represented, accounting for nearly $83 \%$ of OTH clients despite census data indicating $46 \%$ of DC residents are Black or African American [18]. Additionally, 13\% of clients had never been tested, further suggesting that this model reaches high-risk individuals who may have otherwise not received screening. Individuals who identify as White were less likely to have ever been tested for HIV than were those of other racial backgrounds (Table 3). This variance in HIV testing may be due to either self-perceived risk of HIV and the need for testing or may reflect testing initiatives that target groups typically considered to be at higher risk.

A surprising finding from these data was the association between race and HIV risk factors. While Black or African American individuals are among the highest risk groups for new HIV infection in Washington, DC [45], they were less likely to report risk factors than clients of other racial backgrounds. We found that Whites were more likely to report risk factors (Table 3). These findings further emphasize the role of social inequities and structural forces that drive the HIV epidemic. There are many factors, such as residential segregation, social capital, stigma, and psychological influences, that intertwine to explain why Black or African Americans are disproportionately affected by this epidemic [6, 19]. As supported by our findings, lifestyle choices alone do not explain racial inequalities in HIV disease burden, and thus, may be insufficient to appropriately gauge a patient's true HIV risk.

\section{Efficacy and cost}

This program found that $0.47 \%$ of 846 clients screened newly positive for HIV. With an estimated 51.1 new cases per 100,000 DC residents each year ( $0.05 \%)$, and many of the approximately $2 \%-3 \%$ of DC residents living with HIV already linked to care, we believe this rate of new positive screens supports the utility of the program $[2,5]$. To further contextualize this data, OTH found a new positive rate of 227 cases per 100,000 residents per year, more than four times higher than the new positive rate found by traditional testing methods in DC, suggesting value added to a healthcare system that seeks to identify undiagnosed, difficultto-reach cases. Of the six clients who screened positive, two were successfully reached for a 12-month follow-up; one reported successful initial LTC but had since not been retained in care, while the other client was retained in care. The small number of clients who screened positive prohibits further meaningful statistical analysis. The 846 screens and 644 PrEP education sessions were achieved at an estimated operational cost of $\$ 138,367$ over the 25 months of the study period. While a cost-effectiveness analysis is beyond the scope of this work, non-market benefits (e.g., community engagement, the potential for stigma and risk reduction, PrEP education and referral, and knowledge of HIV status) confer added value to this program.

\section{PrEP education and linkage}

Although PrEP is a mainstay of HIV prevention [20], there are significant concerns regarding the need for novel methods of PrEP delivery [21-23], and healthcare providers' limited knowledge of PrEP may be a contributor to reduced uptake [24]. Accordingly, less than $30 \%$ of our clients had ever heard of PrEP, and only $0.88 \%$ were taking PrEP. After a brief education, $27.36 \%$ of the clients requested a same-day PrEP prescription, though a smaller proportion indicated risk factors suggesting an indication for a prescription. The observed low use of PrEP, despite risk factors, correlates well with the extant literature, and this pop-up model may be well-designed to reduce the gap both in education and prescription. Further data regarding PrEP uptake and long-term adherence from this program are needed.

\section{Program feasibility strengths}

While community-based screening comes in a variety of formats, brick-and-mortar clinics and mobile units can be limited in efficacy by spatiotemporal requirements, operational costs, and staffing needs. This rapid pop-up model is designed to reduce such barriers as follows: 
Traditional clinics are often subject to limited hours of operation and are not always located in the highestrisk areas of DC. Some clinics only have HIV screening and LTC services at certain hours of the day, while others may have prohibitively long wait times. All such clinics require individuals to know their physical locations and to take the initiative to seek out their services. Individuals unaware of their risk factors or their preventative care needs maybe even less likely to seek care. Regarding mobile vans, these are conceivably difficult to park in public spaces that have the highest foot traffic.

The pop-up tent model allows for screening on weekends and at multiple locations throughout the city where mobile units might not typically have the space for operations. OTH targets areas, such as grocery stores, pharmacies, and laundromats, where visibility reduces the burden on individuals to seek care.

Operational Costs

OTH spent $\$ 138,367$ on operations over 25 months of screening or approximately $\$ 66,416$ per year, a significantly lower cost of operation than a typical brick-and-mortar clinic or even a mobile van screening program [16]. The use of a tent, which costs approximately $\$ 500$, is a major factor in the cost reduction. Screening kits were provided free of charge by the DC Department of Health and thus do not factor into the operational cost. Expenses such as insurance, licensing, equipment, and others are included in operations costs.

Staffing

Staffing models influence both feasibility and operational costs. The screening kit used by OTH is under CLIA-waived status, and as the operational focus is specifically HIV screening and PrEP education, clinicians are not required on-site. PrEP prescription and other medical care are instead provided by clinical partners during referral or LTC. Screening sessions are thus performed by volunteers who are trained with knowledge and skills to provide rapid HIV screening and PrEP education, risk factor assessment, and LTC. These nonclinical volunteers can also enhance their engagement by serving in leadership and education roles. As community engagement is considered a critical component of improving outcomes for PLWH [25], this unique model contributes to community engagement around HIV and provides an opportunity for young adults to engage in preventative HIV services.

Reducing Stigma

It is difficult to measure how an HIV screening program might directly reduce the stigma surrounding HIV and HIV screening. Fear and misunderstanding about transmission may be a driver of stigma, perhaps a relic of early misconceptions of HIV. For this reason, OTH opted to make education about HIV and PrEP a centerpiece of the program, a decision that may help reduce bias and misconception. The visibility of the program, in open spaces available to the public as opposed to behind closed doors of clinics, may also contribute to normalizing HIV screening and other preventative health measures. A small minority of persons who underwent intake ultimately chose not to receive HIV screening ( $\mathrm{n}=37,4.19 \%)$. While no data are available regarding their decision to forego screening, a variety of factors, such as wait time or anxiety about results, could act as deterrents.

\section{Limitations}

Several limitations are worth noting. LTC and lost to follow-up (LTFU) are established challenges of many organizations, with as much as one-third LTFU at 12 months and more than half not retained in care [26]. Many factors likely contribute to LTFU, including limited transportation, time restrictions, insurance needs, anxiety regarding HIV diagnosis, relocation, and dissatisfaction with a provider. While the timely receipt of screening results (an area where the OTH model excels) is associated with LTC [27], there is significant room for improvement in successful LTC. The benchmark for this metric does not appear to be established, and there was a small total number of OTH clients who screened positive. However, replication of this model may benefit from incorporating additional LTC resources, such as patient navigators or case managers. Additionally, while limited on-site staffing and other resources may reduce throughput time and increase client satisfaction, this is coupled with the drawback of limited opportunities to address other medical concerns, provide on-site confirmatory testing, or initiate treatment. Furthermore, while the INSTI ${ }^{\circledR}$ HIV 1/2 Rapid Antibody Kit is reported to be very accurate [28], there is limited data on the final confirmatory testing of OTH clients who screened positive. Finally, while the pop-up tent model has the benefit of markedly reduced operation costs, data are not available for a cost-effectiveness analysis at this time.

\section{Conclusions}

The OTH pop-up model is a feasible and effective way of increasing community-based HIV screening and identifying a higher rate of newly positive HIV cases than traditional testing methods in the District of Columbia. By reducing access barriers, stigma, and costs, the OTH model provides a unique and needed platform for improving community engagement, HIV education, risk reduction strategies, and knowledge and prescription of PrEP. Organizations with a wealth of resources and staff may benefit from the judicious application of those resources to their community-based screening, as these may decrease efficiency and patient satisfaction. However, after the conclusion of the pilot program reported here, this model was later adapted to include a COVID-19 screening program, alongside HIV screening, which did require an increased 
number of staff members. LTC remains a challenge in pop-up HIV screening, but organizations should maintain a clear LTC plan and same-day LTC should be prioritized. Patient navigators and enhanced patient contact should also be considered, as these improve retention in care in a variety of settings, and may also be beneficial when implementing a pop-up model. We find that this healthcare delivery model reaches critical populations with risk factors for HIV and can be easily replicated to provide other health maintenance activities, which may be important during both the COVID-19 pandemic and the ongoing global HIV epidemic.

\section{Additional Information \\ Disclosures}

Human subjects: Consent was obtained or waived by all participants in this study. Mass General Brigham Institutional Review Board issued approval 2019P003138. Animal subjects: All authors have confirmed that this study did not involve animal subjects or tissue. Conflicts of interest: In compliance with the ICMJE uniform disclosure form, all authors declare the following: Payment/services info: All authors have declared that no financial support was received from any organization for the submitted work. Financial relationships: David Schaffer and Lindsey Sawczuk declare(s) employment from One Tent Health. David Schaffer and Lindsey Sawczuk have previously received salary for their employment by One Tent Health. Other relationships: All authors have declared that there are no other relationships or activities that could appear to have influenced the submitted work.

\section{Acknowledgements}

The authors would like to acknowledge Mackenzie Copley, co-founder of One Tent Health, without whom this program and research could not be possible.

\section{References}

1. Cohen MS, Chen YQ, McCauley M, et al.: Prevention of HIV-1 infection with early antiretroviral therapy . N Engl J Med. 2011, 365:493-505. 10.1056/NEJMoa1105243

2. Estimated HIV incidence and prevalence in the United States, 2010-2016 . (2019). Accessed: December 20, 2021: http://www.cdc.gov/hiv/pdf/library/reports/surveillance/cdc-hiv-surveillance-supplemental-reportvol-24-1.pdf.

3. Consolidated Guidelines on HIV Testing Services for a Changing Epidemic . (2019). Accessed: December 20, 2021: http://www.who.int/publications/i/item/WHO-CDS-HIV-19.31.

4. DC Department of Health HIV/AIDS, Hepatitis, STD and TA: Annual epidemiology \& surveillance report. 2019.. (2019).

http://dchealth.dc.gov/sites/default/files/dc/sites/doh/publication/attachments/2019\%20Annual\%20Surveillance\%20Report

5. The HIV/AIDS Epidemic in Washington, D.C. (2012). Accessed: December 20, 2021: http://www.kff.org/wpcontent/uploads/2013/01/8335.pdf.

6. HIV/AIDS Inequality: Structural Barriers to Prevention, Treatment, and Care in Communities of Color. Why We Need A Holistic Approach to Eliminate Racial Disparities in HIV/AIDS . (2012). Accessed: December 20, 2021: http://www.americanprogress.org/issues/lgbt/reports/2012/07/27/11834/hivaids-inequalitystructural-barriers-to-preven....

7. D.C. Reports Sharp Decline in New HIV Infections . (2017). Accessed: December 20, 2021: https://www.washingtonpost.com/local/dc-politics/dc-reports-sharp-decline-in-new-hivinfections/2017/06/27/60d4ea38-5....

8. Lifetime Risk of HIV Diagnosis . (2016). Accessed: December 20, 2021: http://www.cdc.gov/nchhstp/newsroom/2016/croi-press-release-risk.html.

9. Owens DK, Davidson KW, Krist AH, et al.: Screening for HIV infection: US Preventive Services Task Force recommendation statement. JAMA. 2019, 321:2326-36. 10.1001/jama.2019.6587

10. Krueger A, Van Handel M, Dietz PM, Williams WO, Patel D, Johnson AS: HIV testing, access to HIV-related services, and late-stage HIV diagnoses across US States, 2013-2016. Am J Public Health. 2019, 109:1589-95. 10.2105/AJPH.2019.305273

11. Lipsitz MC, Segura ER, Castro JL, et al.: Bringing testing to the people - benefits of mobile unit HIV/syphilis testing in Lima, Peru, 2007-2009. Int J STD AIDS. 2014, 25:325-31. 10.1177/0956462413507443

12. Hoyos J, Belza MJ, Fernández-Balbuena S, Rosales-Statkus ME, Pulido J, de la Fuente L; Madrid Rapid HIV Testing Group: Preferred HIV testing services and programme characteristics among clients of a rapid HIV testing programme. BMC Public Health. 2013, 13:791. 10.1186/1471-2458-13-791

13. Suthar AB, Ford N, Bachanas PJ, et al.: Towards universal voluntary HIV testing and counselling: a systematic review and meta-analysis of community-based approaches. PLoS Med. 2013, 10:e1001496. 10.1371/journal.pmed.1001496

14. Hellard M, Ryan KE, Stoové M: Point-of-care HIV testing can help achieve UNAIDS targets . Lancet HIV. 2020, 7:e216-17. 10.1016/S2352-3018(20)30043-6

15. Lahuerta M, Sabidó M, Giardina F, et al.: Comparison of users of an HIV/syphilis screening communitybased mobile van and traditional voluntary counselling and testing sites in Guatemala. Sex Transm Infect. 2011, 87:136-40. 10.1136/sti.2010.043067

16. Bassett IV, Govindasamy D, Erlwanger AS, et al.: Mobile HIV screening in Cape Town, South Africa: clinical impact, cost and cost-effectiveness. PLoS One. 2014, 9:e85197. 10.1371/journal.pone.0085197

17. Meulbroek M, Pujol F, Pérez F, et al.: BCN Checkpoint: same-day confirmation of reactive HIV rapid test with point of care HIV-RNA accelerates linkage to care and reduces anxiety. HIV Med. 2018, 19:63-65. 10.1111/hiv.12595

18. QuickFacts: District of Columbia . (2019). Accessed: December 20, 2021: http://www.census.gov/quickfacts/DC. 
19. Pellowski JA, Kalichman SC, Adler N: A pandemic of the poor: social disadvantage and the U.S. HIV epidemic. Am Psychol. 2014, 68:197-209. 10.1037/a0032694.A

20. Preexposure Prophylaxis for the Prevention of HIV Infection in the United States - 2017 Update: A Clinical Practice Guideline. (2018). Accessed: December 20, 2021: http://www.cdc.gov/hiv/pdf/risk/prep/cdc-hivprep-guidelines-2017.pdf.

21. Smith DK, Van Handel M, Grey J: Estimates of adults with indications for HIV pre-exposure prophylaxis by jurisdiction, transmission risk group, and race/ethnicity, United States, 2015. Ann Epidemiol. 2018, 28:85057.e9. 10.1016/j.annepidem.2018.05.003

22. Raymond HF, Snowden JM, Guigayoma J, McFarland W, Chen YH: Community levels of PrEP use among men who have sex with men by race/ethnicity, San Francisco, 2017. AIDS Behav. 2019, 23:2687-93. 10.1007/s10461-019-02428-5

23. Sidebottom D, Ekström AM, Strömdahl S: A systematic review of adherence to oral pre-exposure prophylaxis for HIV - how can we improve uptake and adherence?. BMC Infect Dis. 2018, 18:581. 10.1186/s12879-018-3463-4

24. Mimiaga MJ, White JM, Krakower DS, Biello KB, Mayer KH: Suboptimal awareness and comprehension of published preexposure prophylaxis efficacy results among physicians in Massachusetts. AIDS Care. 2014, 26:684-93. 10.1080/09540121.2013.845289

25. Expanding Your Reach to End the HIV Epidemic: Community Engagement Toolkit . (2012). Accessed: December 20, 2021: http://www.nmac.org/wp-content/uploads/2012/08/NMAC-Community-EngagementToolkit-Web.pdf.

26. Fleishman JA, Yehia BR, Moore RD, Korthuis PT, Gebo KA; HIV Research Network: Establishment, retention, and loss to follow-up in outpatient HIV care. J Acquir Immune Defic Syndr. 2012, 60:249-59. 10.1097/QAI.0b013e318258c696

27. Mugavero MJ, Norton WE, Saag MS: Health care system and policy factors influencing engagement in HIV medical care: piecing together the fragments of a fractured health care delivery system. Clin Infect Dis. 2011, 52:S238-46. 10.1093/cid/ciq048

28. INSTI HIV-1/HIV-2 Antibody Test. (2020). Accessed: December 20, 2021: http://www.fda.gov/vaccinesblood-biologics/approved-blood-products/insti-hiv-1hiv-2-antibody-test. 